\title{
HYDROGEOLOGY OF NIKSAR BASIN, TOKAT, TURKEY
}

\section{DR. SYED MOBASHER AFTAB, PG}

\author{
BALOCHISTAN UNIVERSITY OF INFORMATION \\ TECHNOLOGY, ENGINEERING \& \\ MANAGEMENT SCIENCES, \\ QUETTA, PAKISTAN
}

GEOLOGICAL SOCIETY OF AMERICA, ANNUAL MEETING 2017, SEATTLE, WA, USA SESSION NO. 121; RECENT ADVANCES IN HYDROGEOLOGY, 23 OCTOBER 2017 


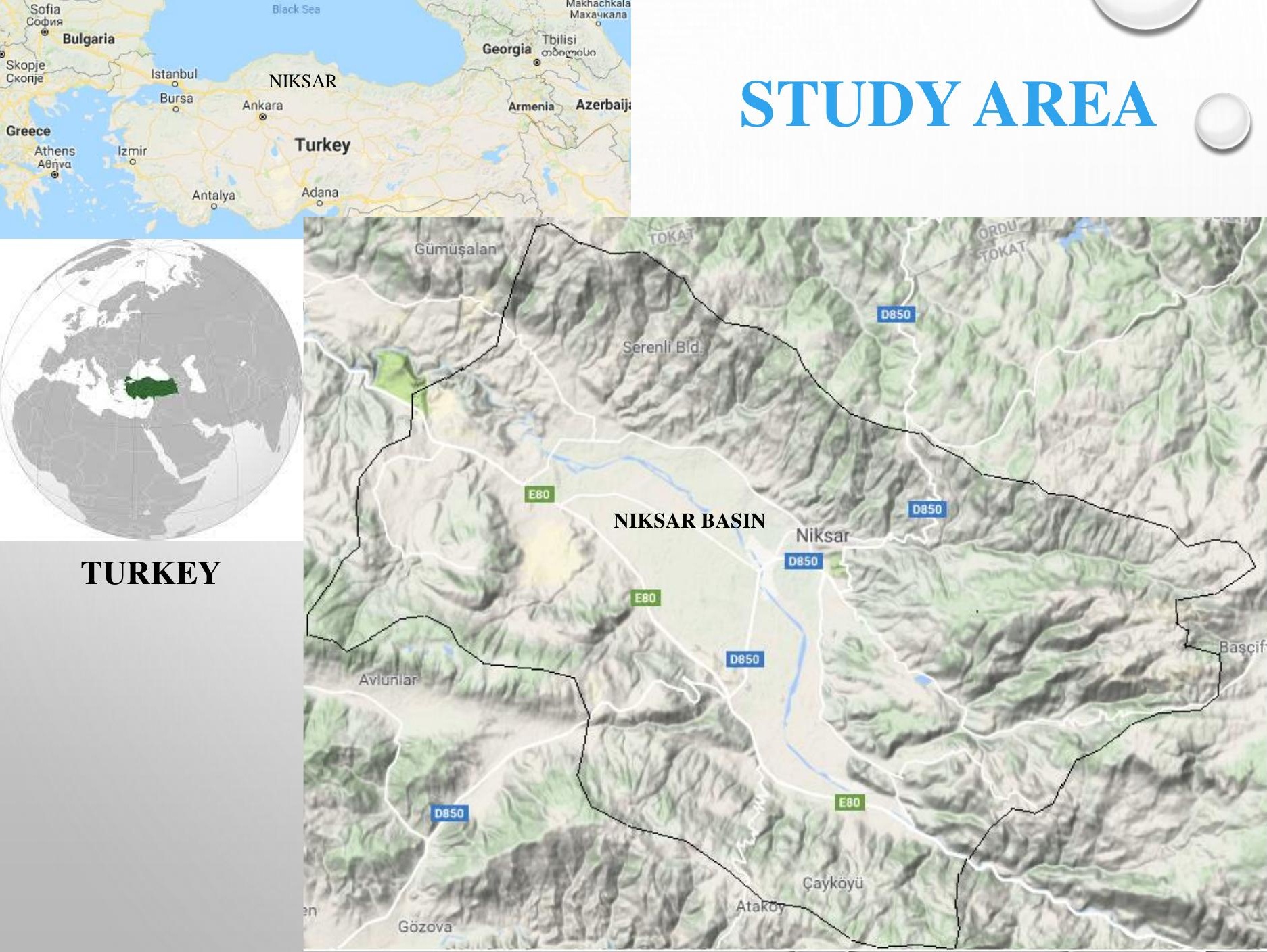




\section{OBJECTIVES}

To study the Geology and Hydrogeology of all Basinal Formations from Paleozoic to Quaternary

$>$ To estimate the recharge and discharge areas and quantities of hydrogeological units

$>$ To evaluate soil water budget by utilizing climatic data

$>$ To monitor the discharge of major karstic springs and estimate storage capacities

$>$ To prepare a physical hydrogeological model of the Niksar Valley aquifer

$>$ To calculate the groundwater budget of the valley - fill aquifer 


\section{HYDROGEOLOGY}

$>$ Lithological units exposed in northern and southern part of Kelkit River grouped as Pontid and Anatolit

$>$ These groups are composed of eleven to four formations and ranged from Paleozoic to Quaternary

$>$ Metamorphic rocks form basement in both groups

$>$ Formations of similar hydrogeological characteristics grouped as permeable, semi-permeable \& impermeable

$>$ Micritic limestone of U Jurassic - L Cretaceous and detrital limestone of $\mathbf{U}$ Cretaceous - L Paleocene form karstic aquifers

$>$ Presence of clay lenses in Pliocene and Quaternary sediments created suitable hydrodynamic conditions for the formation of a confined aguifer. 


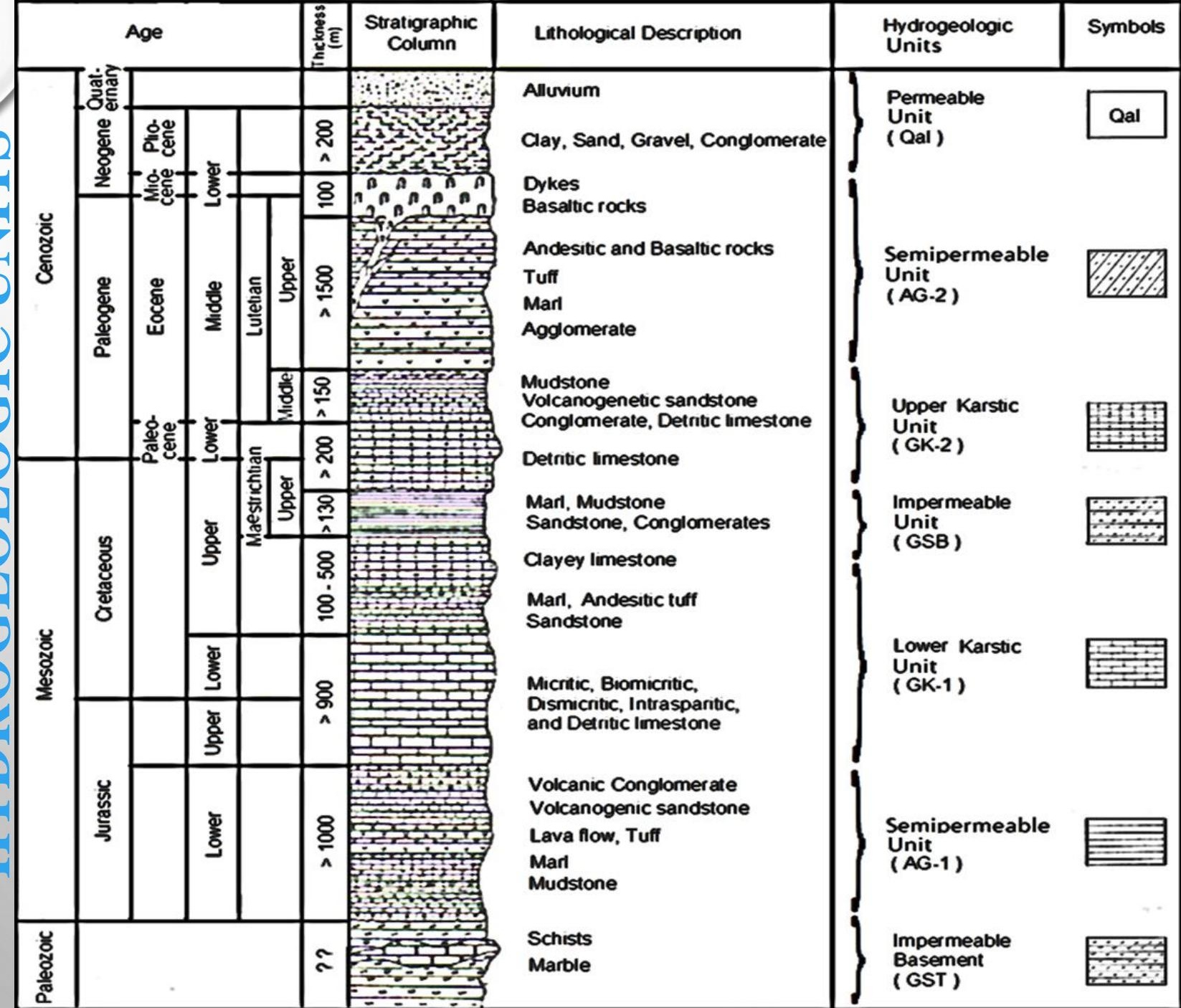




\section{HYDROGEOLOGICAL MAP}

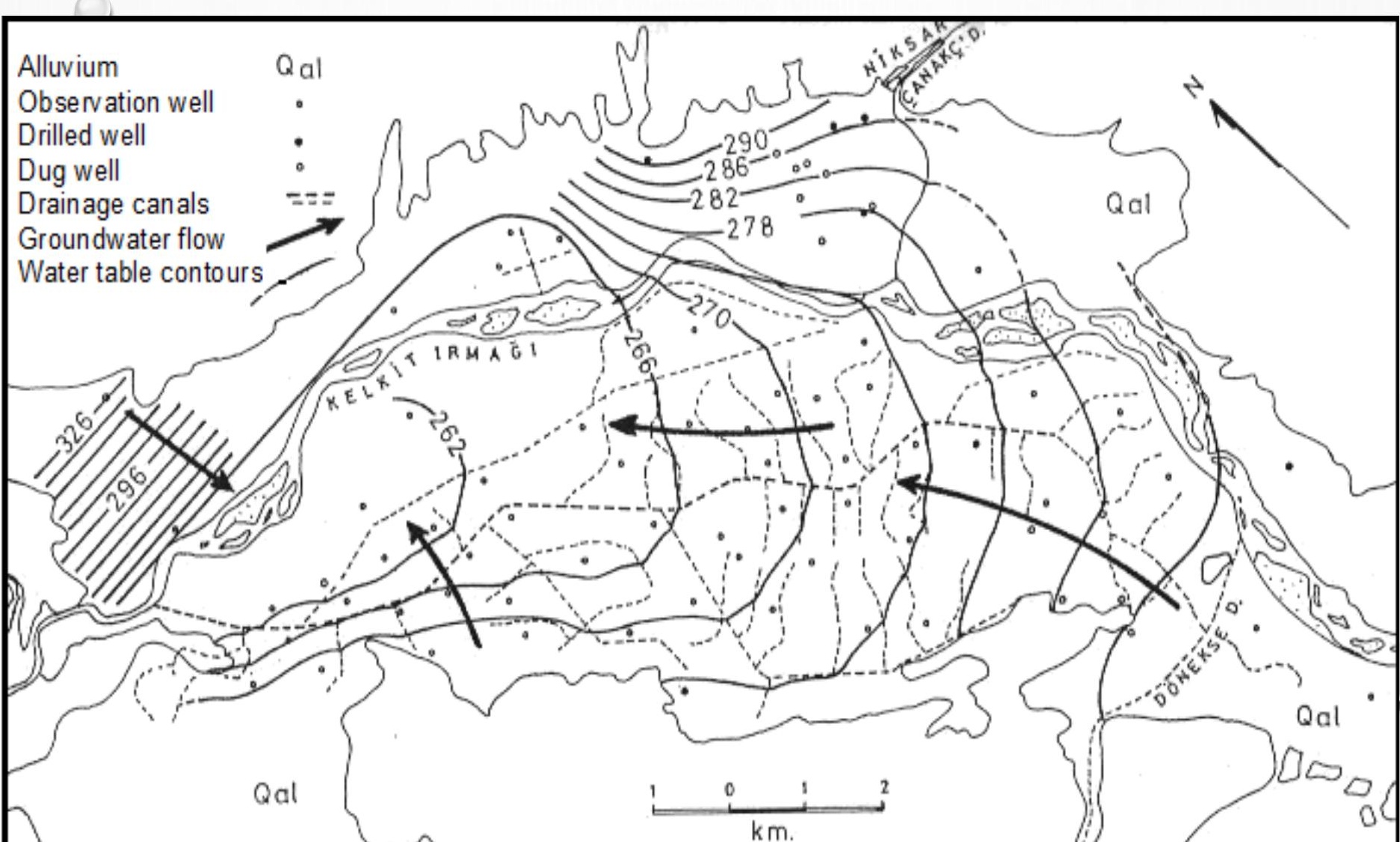




\begin{tabular}{|c|c|c|c|c|c|c|c|c|c|c|c|c|c|}
\hline Budget Elements & Oct & Nov & Dec & Jan & Feb & Mar & Apr & May & Jun & Jul & Aug & Sep $\mid$ & Total \\
\hline Monthly Temp. Co & 14.2 & 6.7 & 5.4 & 6.1 & 7.6 & 5.3 & 11.5 & 18.7 & 20.4 & 23.5 & 21.3 & 19.1 & - \\
\hline Thermal Index (i) & 4.9 & 1.6 & 1.1 & 1.4 & 1.9 & 1.1 & 3.5 & 7.4 & 8.4 & 10.4 & 9.0 & 7.6 & 58.2 \\
\hline Etp (mm) & 55.8 & 19.5 & 14.4 & 17.1 & 23.3 & 14.1 & 41.6 & 82.1 & 92.7 & 113 & 99.0 & 84.6 & 656.7 \\
\hline $\begin{array}{l}\text { Latitude Correction } \\
\text { Factor }\left(40^{0}-3^{0}\right)\end{array}$ & 1.0 & 0.8 & 0.8 & 0.8 & 0.8 & 1.0 & 1.1 & 1.2 & 1.3 & 1.3 & 1.2 & 1.0 & - \\
\hline Etpc (mm) & 53.6 & 16.9 & 11.7 & 14.4 & 19.3 & 14.5 & 46.1 & 102 & 115.9 & 144 & 116 & 88.0 & 741.9 \\
\hline Precipitation (mm) & 26.5 & 66.7 & 78.4 & 77.9 & 32.5 & 73.5 & 87.0 & 27.8 & 41.6 & 15.6 & 23.4 & 0.0 & 550.9 \\
\hline Etr (mm) & 26.5 & 16.2 & 11.7 & 14.4 & 19.3 & 14.5 & 46.1 & 102 & 67.6 & 15.6 & 23.4 & 0.0 & 357.1 \\
\hline Utilization (mm) & 0.0 & 50.5 & 100 & 100 & 100 & 100 & 100 & 26.0 & 0.0 & 0.0 & 0.0 & 0.0 & - \\
\hline Surplus (mm) & 0.0 & 0.0 & 17.2 & 63.5 & 13.2 & 59.0 & 40.9 & 0.0 & 0.0 & 0.0 & 0.0 & 0.0 & 193.9 \\
\hline Deficit (mm) & 27.1 & 0.0 & 0.0 & 0.0 & 0.0 & 0.0 & 0.0 & 0.0 & 48.3 & 128.0 & 92.8 & 88.0 & 384.2 \\
\hline Runoff (mm) & 0.0 & 0.0 & 8.6 & 36.1 & 24.6 & 41.8 & 41.4 & 0.0 & 0.0 & 0.0 & 0.0 & 0.0 & 152.5 \\
\hline
\end{tabular}




\section{MIONITORING WELLS}

\begin{tabular}{|c|c|c|c|c|}
\hline $\begin{array}{c}\text { Monitoring } \\
\text { Wells }\end{array}$ & No. & Hydrogeologic Unit & Depth (m) & Discharge (1/s) \\
\hline Dug Wells & 13 & Alluvium & $5-23$ & - \\
\hline Old Piezometers & 48 & Alluvium & $1.2-3.4$ & - \\
\hline New Piezometers & 15 & Alluvium & $3-4.5$ & - \\
\hline \multirow{3}{*}{ Drilled Wells } & 4 & Alluvium & $18-110$ & $5-10$ \\
\hline & 2 & Limestone & $25-85$ & 5 \\
\hline & 3 & Pliocene & $118-200$ & $4-30$ \\
\hline Artesian Wells & 10 & Pliocene & $20-240$ & $30-63$ \\
\hline
\end{tabular}


CORRELATIOX; PRECIPITATION, RIVER FLOW, ARTYSUAN WELJDISCHCARGE \& GROUNDWATER FLUCTUATIONS

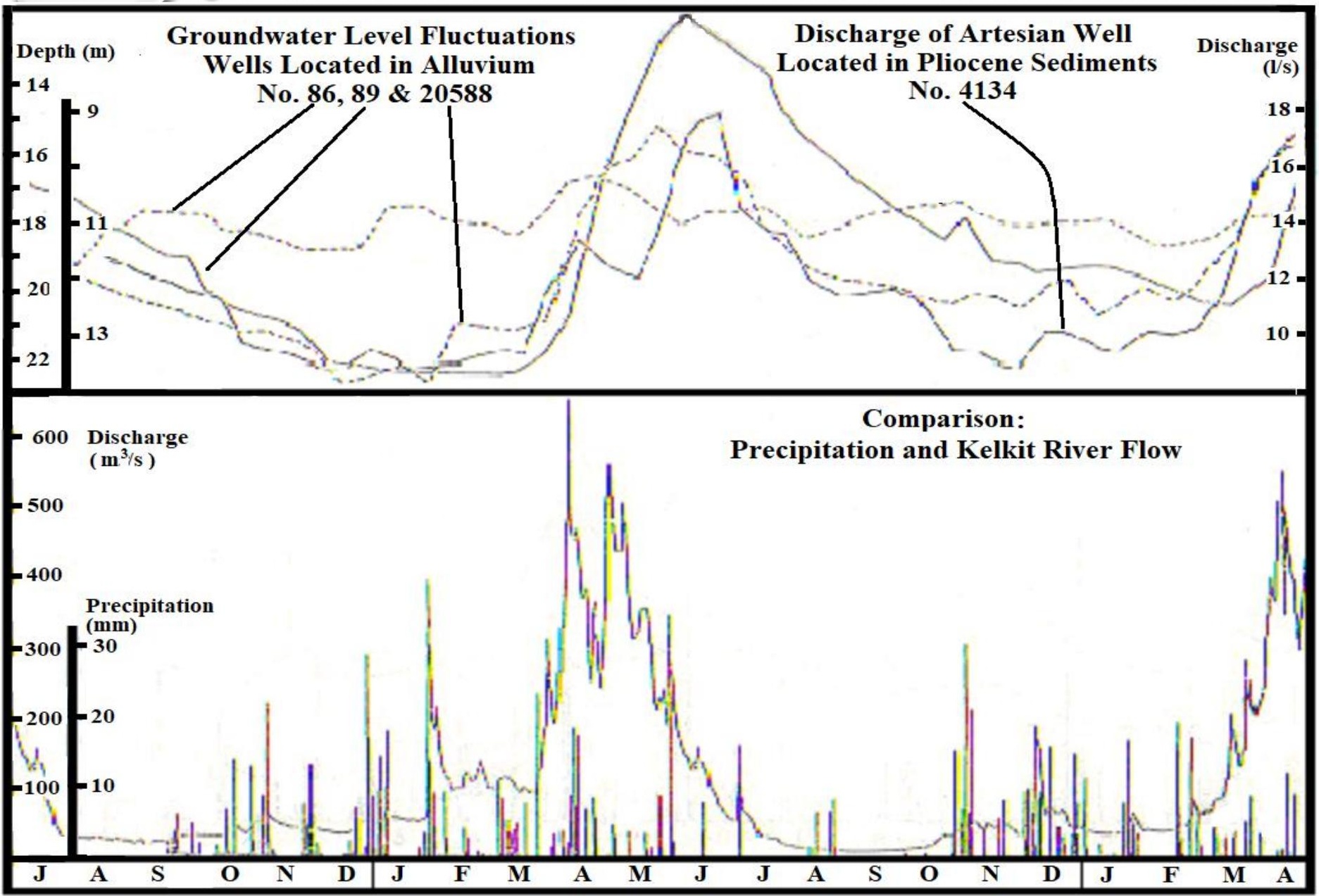


WELL PUMPING TEST ANALYSIS

\begin{tabular}{|c|c|c|c|c|c|c|c|}
\hline \multirow{2}{*}{$\begin{array}{l}\text { Well } \\
\text { No. }\end{array}$} & \multirow{2}{*}{$\begin{array}{c}\text { Lithological } \\
\text { Unit }\end{array}$} & \multicolumn{2}{|c|}{ Thies } & \multicolumn{2}{|c|}{ Jacob } & \multicolumn{2}{|c|}{ Recovery } \\
\hline & & $\mathbf{T}\left(\mathrm{m}^{2} / \mathrm{s}\right)$ & $\mathbf{K}(\mathbf{m} / \mathbf{s})$ & $\mathrm{T}\left(\mathrm{m}^{2} / \mathrm{s}\right)$ & $K(\mathbf{m} / \mathbf{s})$ & $\mathbf{T}\left(\mathrm{m}^{2} / \mathrm{s}\right)$ & $\mathbf{K}(\mathbf{m} / \mathbf{s})$ \\
\hline 27291 & Alluvium & $1.4 \times 10^{-3}$ & $1.0 \times 10^{-4}$ & $1.1 \times 10^{-3}$ & $7.9 \times 10^{-5}$ & - & - \\
\hline 20588 & Alluvium & $3.2 \times 10^{-2}$ & $2.1 \times 10^{-3}$ & $7.2 \times 10^{-2}$ & $4.8 \times 10^{-3}$ & - & - \\
\hline 20159 & Alluvium & $1.35 \times 10^{-3}$ & $7.5 \times 10^{-5}$ & $4.8 \times 10^{-3}$ & $2.6 \times 10^{-4}$ & - & - \\
\hline 20159 & Alluvium & $1.34 \times 10^{-3}$ & $7.4 \times 10^{-5}$ & $2.3 \times 10^{-3}$ & $1.3 \times 10^{-4}$ & $7.9 \times 10^{-3}$ & $4.4 \times 10^{-4}$ \\
\hline 20158 & Alluvium & $5.3 \times 10^{-3}$ & $4.4 \times 10^{-4}$ & $5.9 \times 10^{-3}$ & $4.9 \times 10^{-4}$ & - & - \\
\hline 27267 & Pliocene & $5.2 \times 10^{-5}$ & $3.0 \times 10^{-6}$ & $5.4 \times 10^{-5}$ & $3.2 \times 10^{-6}$ & $9.0 \times 10^{-5}$ & $5.3 \times 10^{-6}$ \\
\hline 4985 & Pliocene & $2.6 \times 10^{-4}$ & $5.3 \times 10^{-6}$ & $5.1 \times 10^{-4}$ & $1.0 \times 10^{-5}$ & - & - \\
\hline 4136 & Pliocene & $1.4 \times 10^{-3}$ & $1.3 \times 10^{-5}$ & $4.3 \times 10^{-3}$ & $4.1 \times 10^{-5}$ & - & - \\
\hline 4684 & Limestone & $1.74 \times 10^{-3}$ & - & - & - & - & - \\
\hline SK-2 & Limestone & - & $5.2 \times 10^{-5}$ & - & - & - & - \\
\hline SK-3 & Limestone & - & $5.2 \times 10^{-5}$ & - & - & - & - \\
\hline
\end{tabular}




\section{SLUG TEST ANALYSIS}

Well No.

64

65

66

67

68

69

70

71

72

73

74

75

76
$\mathrm{T}\left(\mathrm{m}^{2} / \mathrm{s}\right)$

$1.1 \times 10^{-5}$

$5.2 \times 10^{-6}$

$9.0 \times 10^{-5}$

$4.0 \times 10^{-6}$

$6.9 \times 10^{-6}$

$3.0 \times 10^{-5}$

$3.1 \times 10^{-5}$

$1.3 \times 10^{-6}$

$3.0 \times 10^{-5}$

$3.3 \times 10^{-6}$

$2.4 \times 10^{-5}$

$3.2 \times 10^{-5}$

$3.9 \times 10^{-5}$
$\mathrm{K}(\mathrm{m} / \mathrm{s})$

$9.17 \times 10^{-6}$

$3.4 \times 10^{-6}$

$5.2 \times 10^{-5}$

$3.5 \times 10^{-6}$

$3.1 \times 10^{-6}$

$1.1 \times 10^{-5}$

$2.1 \times 10^{-5}$

$7.8 \times 10^{-7}$

$1.5 \times 10^{-5}$

$2.1 \times 10^{-6}$

$1.7 \times 10^{-5}$

$1.9 \times 10^{-5}$

$1.4 \times 10^{-5}$
S

$5.6 \times 10^{-4}$

$5.6 \times 10^{-3}$

$2.5 \times 10^{-3}$

$2.5 \times 10^{-5}$

$2.5 \times 10^{-2}$

$3.6 \times 10^{-4}$

$2.2 \times 10^{-3}$

$5.6 \times 10^{-3}$

$2.5 \times 10^{-3}$

$3.6 \times 10^{-3}$

$2.5 \times 10^{-4}$

$2.5 \times 10^{-2}$

$3.6 \times 10^{-2}$ 


\section{RECHXRGE AND DISCHARGE OF HYDROGEOLOGICAL UNITS}

\section{Hydrogeological Units}

\section{Permeable (Qal)}

Semipermeable (AG-3)

Semipermeable (AG-2)

Upper - Karstic (GK-2)

Impermeable (GSB)

Lower - Karstic (GK-1)

Semipermeable (AG-1)

Impermeable Basement (GST)

Total
Area

$\left(\mathrm{Km}^{2}\right)$

141

120

134

16

15

108

61

59

655
Recharge

$\left(10^{9} \mathrm{~m}^{3}\right)$

2.660

0.005

0.006

0.006

0.001

0.043

0.002

0.001

2.724
Discharge

$\left(10^{9} \mathrm{~m}^{3}\right)$

2.630

0.003

0.002

0.009

0.001

0.036

0.001

0.001

2.683 
$>$ A physical model of Niksar Valley prepared with realistic field conditions, model signifies hydroclimatic and hydrogeological data.

$>$ Input parameters of model are temperature, precipitation, humidity, thermal index and wind direction with associated groundwater recharge.

$>$ Land cover parameters are depth of topsoil, porosity, permeability and residual water content.

$>$ Model signify topography, hydraulic-head conditions, storability, transmissivity \& hydraulic conductivity

$>$ The actual evapotranspiration, surface runoff and groundwater discharge are resultant parameters. 


\section{HYYDOGEOLOGICAL MODEL}

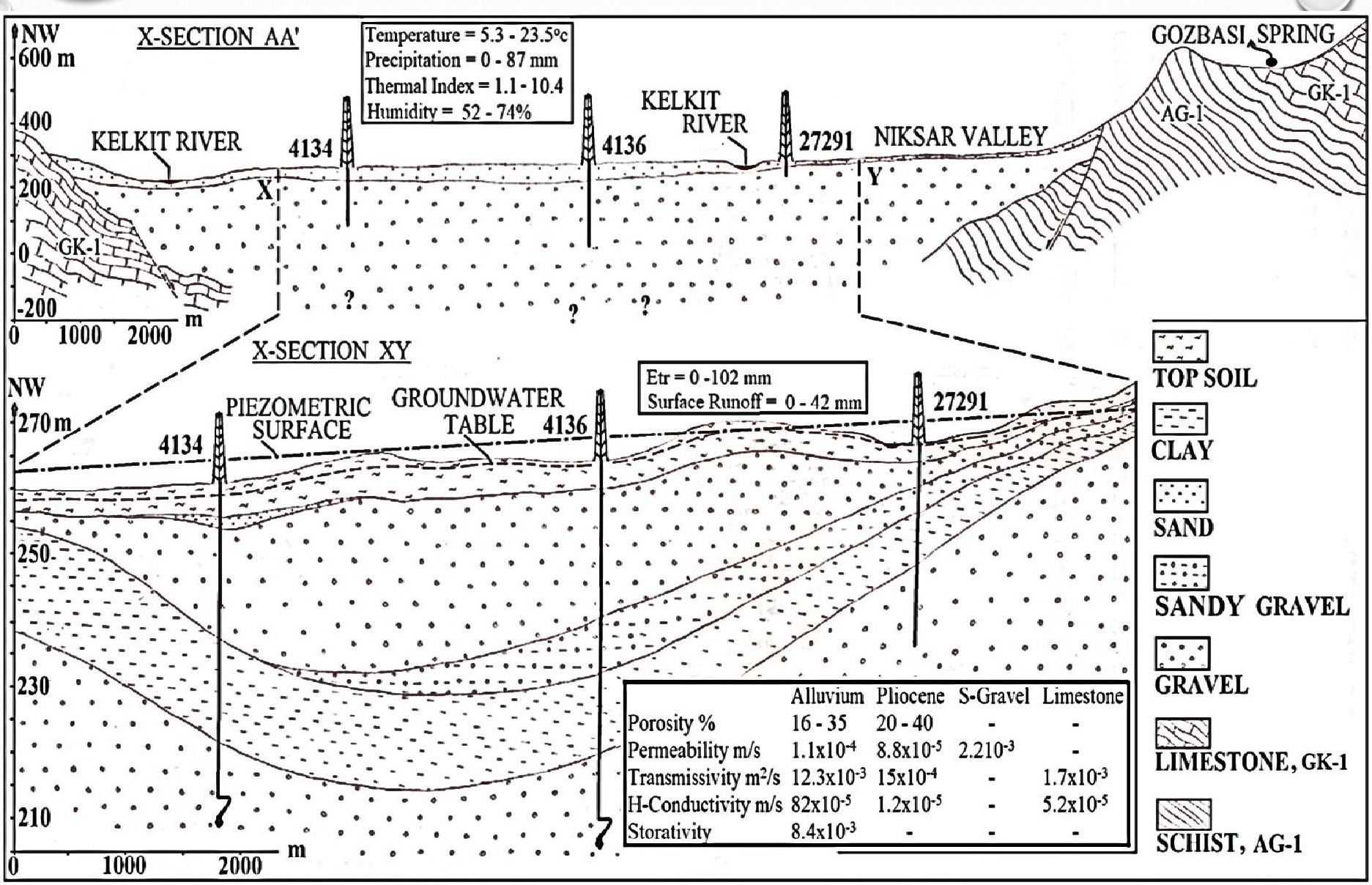




\section{GROUNDWATER BUDGET OF QUATERNARY AQUIFER}

Sources of Recharge

Volume $10^{9} \mathrm{~m}^{3}$

Sources of Discharge

Volume $10^{9} \mathrm{~m}^{3}$

Precipitation

Kelkit River

Canakci Stream

Adjacent Aquifers

Irrigation Waters

Total
0.81

1.71

0.01

0.07

0.06

Drainage Canals

Pumping from Wells

Addition to Reserve
0.50

0.19

0.12

0.55

1.25

0.02

2.63 


\section{GROUNDWATER BUDGET ELEMENTS OF QUATERNARY AQUIFER}
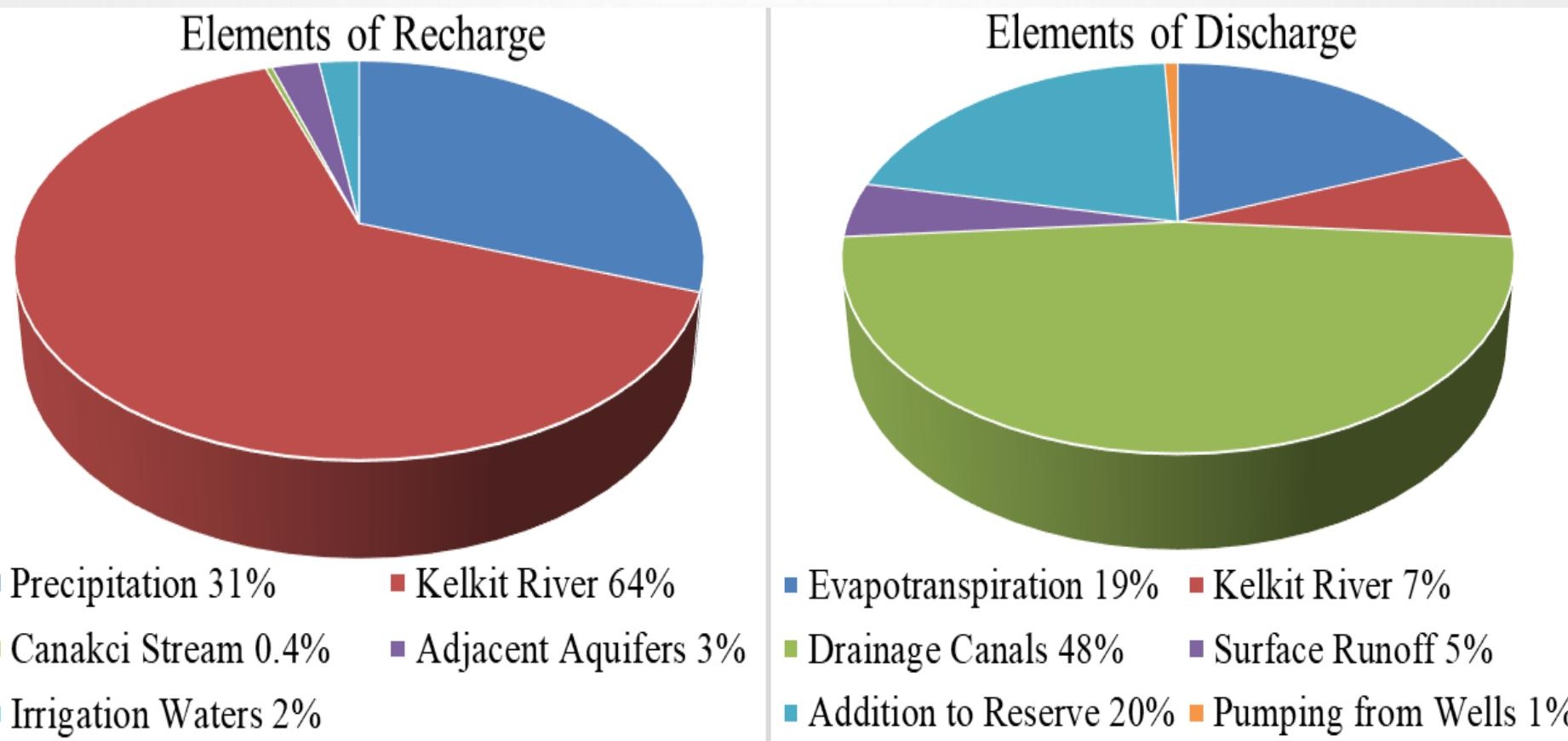


\section{CONCLUSIONS}

> Physical Hydrogeologic Model may translate into GIS based three-dimensional numerical GW flow model

$>$ Discharge of the major karstic spring ranges from 55 $430 \mathrm{l} / \mathrm{s}$ and storage capacity of $0.24 \times 10^{6} \mathrm{~m}^{3}$ to $2.24 \times 10^{6} \mathrm{~m}^{3}$

$>$ During the study period the exposed geological formations recharged through precipitation $2.72 \times 10^{9} \mathrm{~m}^{3}$ and discharged $2.68 \times 10^{9} \mathrm{~m}^{3}$ of groundwater

$>$ Groundwater budget of valley-fill aquifer represents that aquifer received $2.66 \times 10^{9} \mathrm{~m}^{3}$ and discharged $2.63 \times 0^{9} \mathrm{~m}^{3}$ of groundwater 


\section{REFERENCES}

[1] De Martonne, E., 1942. Nouvelle carte mondiale de l'indice d'aridité. Annales de Géographie 51, (French), pp. $242-250$

[2] Thornthwaite, C Wr., 1948. A New and Improved Classification of Climates. Geogr. Rev. 38-1

[3] Turc, L., 1961. Water requirements assessment of irrigation, potential evapotranspiration: Simplified and updated climatic formula. Annales Agronomiques, pp. 12, 13-49

[4] Seymen, I., 1975. 'The Tectonic Features of the North Anatolian Fault Zone in Kelkit Valley", Ph. D. Thesis, ITU Mineral Faculty, Istanbul, (Turkish). pp. 192

[5] Schoeller, H., 1962. Les eaus souterraines. Masson et Cie, Paris, France, pp. 642

[6] E. I. E., 1968-1988.The daily discharge rates data of Kelkit River.

[7] Blumenthal, M M., 1950. Orta ve Asagi Yesilirmak Bolgelerinin (Tokat, Amasya, Havza, Erbaa, Niksar) Jeolojisi Hakinda. M.T.A. Seri D, No.4, (Turkish) Ankara, Turkey

[8] Atkinson, T. C., Hydrol, J., 1977. Diffuse Flow and Conduit Flow in Limestone Terrain in Mendip Hills, Somerset, Great Britain, 35, pp. 93-100.

[9] Gunn, J., G. Giinay \& A. I. Johnson, 1985. A Conceptual Model for Conduit Flow Dominated Karst Aquifers”, in Karst Water Resources. Proc. Ankara Symp. IAHS, Publ. No. 161, pp. 587-596

[10] Castany G., (1963). Traité pratique des eaux souterraines. Dunod, Paris, 658 pp

[11] Schoeller, H., 1967. Methods Pour Obtenir Le Bilan Des Eaux Souterrainex. Extrait de "Eaux Souterraines" Assemblee Generate de Berne, Louvain, Belgium

[12] Theis, C.V., 1935. The relation between the lowering of the piezometric surface and the rate and duration of discharge of a well using groundwater storage, Am. Geophys. Union Trans., vol. 16, pp. 519-524.

[13] Jacob, C.E., 1947. Drawdown test to determine effective radius of artesian well, Trans. Amer. Soc. of Civil Engrs., vol. 112, paper 2321, pp. 1047-1064.

[14] de Marsily, Ghislain, 1986, Quantitative hydrogeology Groundwater hydrology for engineers: New York, Academic Press, Inc., pp. 440

[15] Bouwer, H. H., 1989. The Bower and Rice slug test - An update. Ground Water. v. 27, no. 3, pp. 304-309

[16] D. S. I., and D. M. I., 1968-1988. The monthly drainage, precipitation, temperature and wind speed at different meteorological stations in and around Niksar

[17] Syed, M. A., 1989. Hydrogeological Investigations of Niksar (TOKAT), Valley, Turkey. PhD Dissertation, Unpublished. Ankara University, Turkey, pp.324

[18] Schoeller H (1967). Quantitative evaluation of groundwater resources. In: Methods and Techniques of Groundwater Investigation and Dev. UN Water Resources Series 33: pp. 21-44.

[19] CANIK, B., 1971. Yeralti Suya Rilaneosu, MTA dergisi No. 76, Turkish, Ankara, Turkey

[20] Syed M Aftab, AFSIN, M., and CELIK, M., (1997). Hydrogeological Study and Discharge Features of the Niksar Karst Springs, Turkey. The Arabian Journal for Science and Engineering. Dehran, Saudi Arabia. Vol. 22, No.2A, P. 131-144

[21] Syed M Aftab, (1996). Geotectonics and Depositional History of Niksar (Tokat) Basin, Turkey. Abstracts with Programs, 1996 Annual Meeting, Geological Society of America. October 28-31, 1996. Denver, Colorado USA. P. A-308

[22] CANIK, Baki, and Syed M Aftab, (1990). Groundwater Budget of Niksar (Tokat) Valley, Turkey. "Communication", Journal of the Science Faculty of Ankara University, Turkey. Vol.8,P. 1-11 